\title{
Epidemiology of epithelial ovarian cancer: a tertiary hospital based study in Goa, India
}

\author{
Dweep Jindal, Mrinalini Sahasrabhojanee, Manjusha Jindal*, Joachim D’Souza
}

Department of Obstetrics and Gynecology, Goa Medical college, Bambolim, Goa, India

Received: 04 April 2017

Accepted: 02 May 2017

\section{* Correspondence:}

Dr. Manjusha Jindal,

E-mail: manjushajindal@gmail.com

Copyright: (C) the author(s), publisher and licensee Medip Academy. This is an open-access article distributed under the terms of the Creative Commons Attribution Non-Commercial License, which permits unrestricted non-commercial use, distribution, and reproduction in any medium, provided the original work is properly cited.

\begin{abstract}
Background: Ovarian malignancy is seventh most common cancer in women globally. With increase in longevity, the incidence of epithelial ovarian cancer is increasing and its etiopathology remains unknown. We present preliminary epidemiological findings to help prioritize research.

Methods: Present epidemiological study is retrospective, descriptive study over two years. The collected data was analyzed using SPSS software for overall survival, with respect to stage of disease and histopathology type. Multivariate analysis was done to know independent risk factors.

Results: 114 cases of primary ovarian epithelial cancer were analyzed. Patients' mean age at diagnosis was $52.1 \pm 8.96$ years (median=52). 52.6\% patients had stage III disease at first visit. Serous adenocarcinoma $(85.05 \%)$ was the most prevalent type of histopathology followed by mucinous $(7.1 \%)$, clear cell $(6.1 \%)$ and Endometroid carcinoma $(1.75 \%)$. Surgery followed by combination of taxane and platinum chemotherapy was first line treatment in $35 \%$. The mean age at diagnosis was more with advancing stage (stage one $44 \pm 9.53$ and stage four $55.35 \pm 9.74$ years) but it was not statistically significant $(\mathrm{p}=0.098)$ Advancing age demonstrated poor survival (log-rank $\mathrm{p}=0.05)$ but survival was not significantly different in relation to histopathology $(\log$-rank $=0.629)$. On multivariate regression analysis age of patient $(\mathrm{p}=0.004)$, and stage of disease $(\mathrm{p}=0.005)$ were found to be independent risk factors while histopathology was not an independent risk factor $(\mathrm{p}=0.688)$ for survival.

Conclusions: Research should be aimed to find tools for screening and early diagnosis as well as better therapeutic approaches for advanced epithelial ovarian cancer.
\end{abstract}

Keywords: Epidemiology, Epithelial ovarian cancer, Ovarian malignancy

\section{INTRODUCTION}

Ovarian malignant tumors have varied clinical and biologic behaviour. It is the sixth most common cancer among women (Age standaradised incidence rate being $6.6 / 100,000$ ) and seventh leading cause of cancer deaths globally (age standaradised mortality rate being 4.0/100,00). ${ }^{1}$ In India, during the period 2004-2005, proportion of ovarian cancer varied from $1.7 \%$ to $8.7 \%$ of all female cancers in various population based registries of Indian Council of Medical Research. The proportion of this cancer was $6.0 \%-7.7 \%$ of all cancers among females in Gujarat. The Age Specific Incidence Rate (ASIR) for ovarian cancer revealed that the disease increases from 35 years of age and reaches a peak between the ages 55-64. ${ }^{2}$ Late presentation and ineffective screening methods are impediments in its early detection. The screening tests in form of estimation of CA-125 and Transvaginal sonography are non-specific. ${ }^{3}$ Therefore, the diagnosis is made in late stage when cure rates are low and with increased morbidity due to limited effective treatment options. Five-year survival rates range from $30-50 \%$ for all 
stages of disease. ${ }^{1}$ Standard treatment protocol for ovarian cancer consists of cyto-reduction followed by adjuvant combination chemotherapy consisting of platinum and taxol group of drugs. But due to detection in late stages of disease the recurrence is common and eventually death follows.

With increasing life expectancy, there is increase in incidence of ovarian cancer making it a public health issue. Therefore, an understanding of epidemiologic and genetic factors of ovarian cancer is important information for public health and health care planning for prevention, screening, early diagnosis and control of disease.

Present study is to present the characteristics of epithelial ovarian cancer (EOC) in patients presenting to Goa Medical college.

\section{METHODS}

Present hospital based epidemiological study of ovarian cancer is retrospective, descriptive type, carried out at Department of Obstetrics and Gynaecology, Goa Medical College, a tertiary care centre from January 2013 to December 2014. 125 cases of Ovarian malignancy were admitted during this period. There were 6 cases of germ cell tumor, 3 cases had Kruckenberg's tumor, 2 cases were mixed sex cord stromal tumor and the majority 114 cases were of epithelial ovarian malignancy. All cases were diagnosed based on clinical findings, supported by imaging consisting of ultrasound, CT scan, and MRI. The diagnosis was confirmed on histopathology. Tumour marker serum CA125 supported the diagnosis and was marker for response to therapy. Study data included age at diagnosis, obstetric history, menstrual history, personal and family history, medical history for risk factors and presenting complaints. FIGO stage of ovarian malignancy, histopathological type and management also was noted. Data was expressed in terms of percentage and means with standard deviation. Statistical relevance was assessed in terms of age, stage of disease and histopathology using multivariate analysis. using one way analysis of variance. Kaplan Meier curves were plotted to understand survival with respect to stage of disease and histopathology type and comparison was done using log rank test. The test was considered significant at probability of 0.05 of $\log$ rank analysis.

\section{RESULTS}

There were 256 cases of genital malignancies among 2112 gynaecological admissions during the two-year period $(12.12 \%)$. There were 125 cases of ovarian malignancy (5.92\% in all admissions, $48.8 \%$ in genital malignancies). Majority of ovarian malignancies were primary epithelial tumors (114) contributing to $5.39 \%$ cases among total admissions and $44.5 \%$ among all genital malignancies. Age: Patient's mean age at diagnosis of epithelial ovarian malignancy was $52.1 \pm 8.96$ (median=52). The mean age at diagnosis for Stage I was $44 \pm 9.53$ (median=43), for Stage
II $46.25 \pm 5.9$ (median=48), 51.9 \pm 8.25 (median=53) for Stage III, and 55.35 \pm 9.74 (median=54) for Stage IV (Table 1).

Table 1: Age wise distribution of epithelial ovarian malignancy at various stages.

\begin{tabular}{|c|c|c|c|}
\hline $\begin{array}{l}\text { Stage } \\
\text { (FIGO) }\end{array}$ & $\begin{array}{l}\text { Total no. of } \\
\text { cases }(n=114)\end{array}$ & Mean age & $\begin{array}{l}\text { Median } \\
\text { age }\end{array}$ \\
\hline Stage I & 6 & $44 \pm 9.53$ & 43 \\
\hline Stage II & 8 & $46.25 \pm 5.9$ & 48 \\
\hline Stage III & 64 & $51.9 \pm 8.5$ & 53 \\
\hline Stage IV & 36 & $55.35 \pm 9.74$ & 54 \\
\hline
\end{tabular}

\section{Parity}

There were twelve (10.5\%) nulliparous patients. They were investigated and treated for infertility. Twenty-two cases were para one, 32 cases were para 2, 24 cases were para 3 and 24 cases had $>3$ parity (Table 2 ).

Table 2: Parity wise distribution of epithelial ovarian malignancy $(n=114)$ cases.

\begin{tabular}{|lll|}
\hline Parity & No. of patients & $\%$ \\
\hline Nulliparous & 12 & 10.5 \\
\hline Para I & 22 & 19.29 \\
\hline Para II & 32 & 28.07 \\
\hline ParaIII & 24 & 21.05 \\
\hline Para $>3$ & 24 & 21.05 \\
\hline
\end{tabular}

\section{Menstrual history}

Seventy-four $(65 \%)$ patients were postmenapausal and $35 \%$ were regularly menstruating.

\section{Family history}

Seven patients $(6.15 \%)$ had family history of epithelial malignancy. One patient's father had Carcinoma Rectum, five patient's mother had Carcinoma Breast and one patient's mother had Lukemia. All seven patients presented in stage III epithelial ovarian malignancy.

\section{Presenting complaint}

Commonest presenting complaint was dyspepsia followed by abdominal distension. The other presenting symptoms are shown in Table 3.

FIGO stage wise distribution: In our study, 5.26\% patients were diagnosed in stage I, $7.01 \%$ in stage II, $56.14 \%$ in stage III while $31.57 \%$ in stage IV. Histopathology of Epithelial ovarian malignancy: Serous adenocarcinoma was commonest Histology subtype and was seen in 97 cases $(85.08 \%)$ followed by clear cell carcinoma $7 \%$, mucinous carcinoma $6.1 \%$ and endometroid carcinoma $1.75 \% .58(50.8 \%)$ patients had poorly differentiated, 48 
(42\%) had moderately differentiated and $8(7 \%)$ had well differentiated malignancy (Table 4).

Table 3: Presenting symptoms in EOC.

\begin{tabular}{|lll|}
\hline Presenting complaint & No of patients & $\%$ \\
\hline $\begin{array}{l}\text { Dyspepsia } \\
\begin{array}{l}\text { Lump in abdomen, } \\
\text { distension }\end{array}\end{array}$ & 102 & 89.47 \\
\hline Pain in abdomen & 40 & 56.14 \\
\hline $\begin{array}{l}\text { Loss of appetite and } \\
\text { weight }\end{array}$ & 40 & 35.08 \\
\hline Postmenapausal bleeding & 6 & 35.08 \\
\hline Urinary complaints & 4 & 5.26 \\
\hline Breathlessness & 14 & 3.5 \\
\hline Nausea, vomiting & 2 & 12.28 \\
\hline $\begin{array}{l}\text { Irregular menstrual } \\
\text { bleeding }\end{array}$ & 12 & 1.75 \\
\hline
\end{tabular}

Table 4: Histopathological distribution.

\begin{tabular}{|lll|}
\hline Histopathological type & No. of patients & $\%$ \\
\hline $\begin{array}{l}\text { Serous } \\
\text { cystadenocarcinoma }\end{array}$ & 97 & 85.08 \\
\hline Clear cell carcinoma & 8 & 7 \\
\hline Mucinous carcinoma & 7 & 6.1 \\
\hline Endometroid carcinoma & 2 & 1.75 \\
\hline Poorly differentiated & 58 & 50.87 \\
\hline Moderately differentiated & 48 & 42.1 \\
\hline Well differentiated & 8 & 7.03 \\
\hline
\end{tabular}

\section{Management}

Forty $(35 \%)$ patients were treated with surgery (TAH with BSO with infracolic omentectomy and lymphadenectomy) followed by chemotherapy, $34(29.82 \%)$ patients had NACT followed by surgery and post-operative chemotherapy, 22 (19.29\%) patients were taking NACT and awaiting surgery, 16 cases (14\%) were given palliative chemotherapy and two patients died after diagnosis before initiation of chemotherapy.

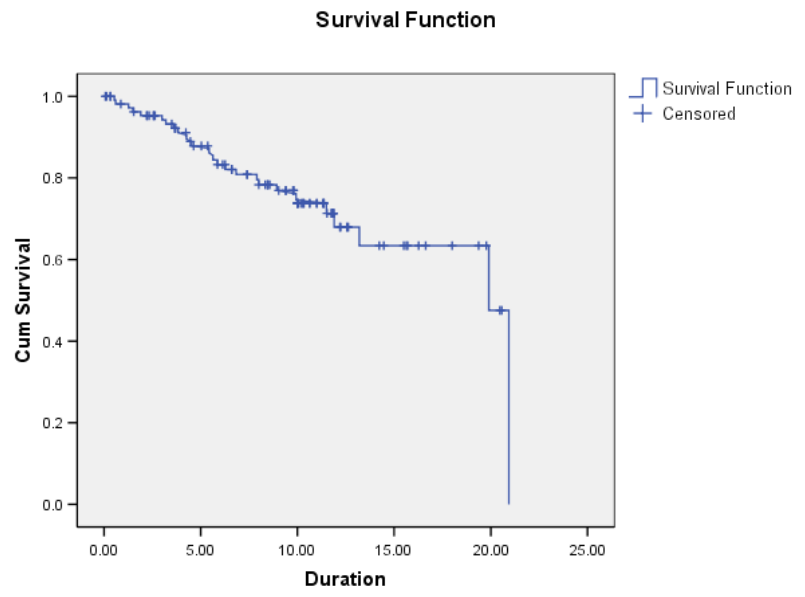

Figure 1: Predicted survival \%age in EOC.
Overall survival as estimated by kaplan Meier curve is as shown in Figure 1. The curves were analyzed in relation to stage and histopathology. Survival was not significantly different in relation to histopathology (log-rank=0.629) Figure 2; though advancing stage demonstrated poor survival (log-rank $\mathrm{p}=0.05$ ) Figure 3 . Further on multivariate regression analysis age of patient $(\mathrm{p}=0.004)$, and stage of disease $(\mathrm{p}=0.005)$ were found to be independent risk factors while histopathology was not an independent risk factor $(\mathrm{p}=0.688)$.

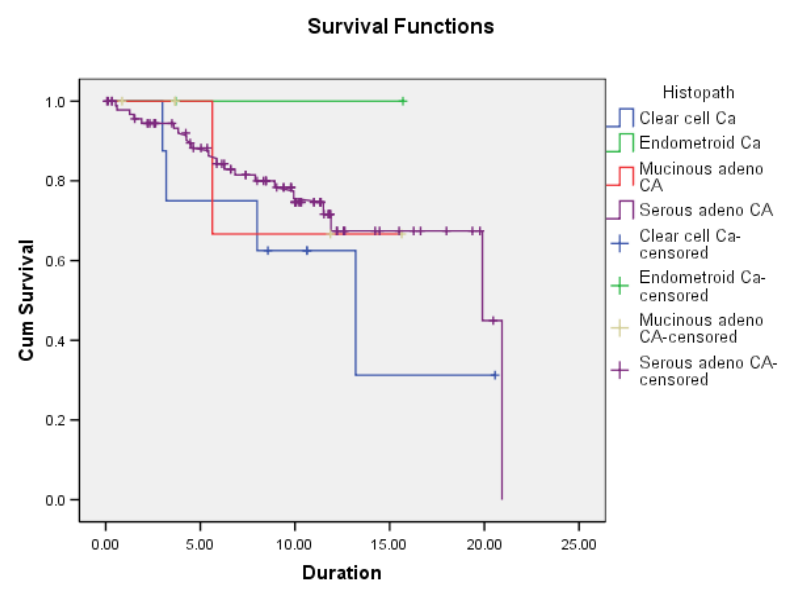

Figure 2: Survival in relation to histology subtype.

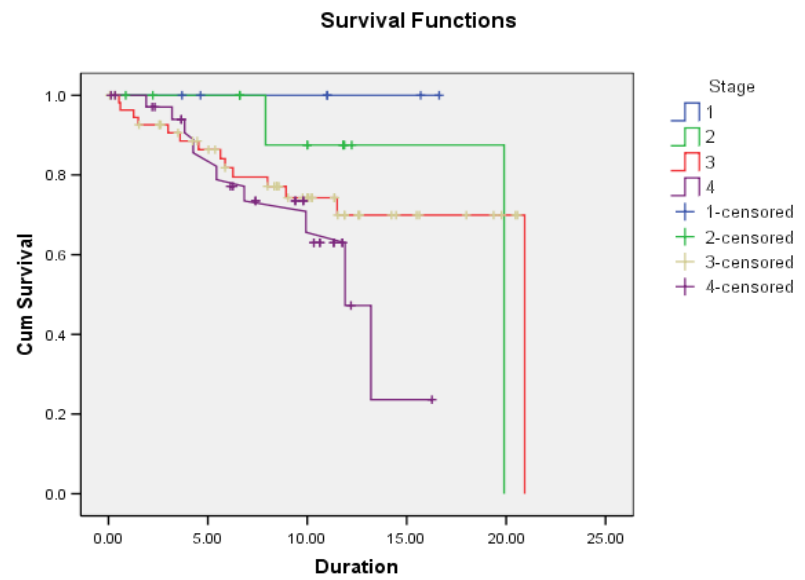

Figure 3: Survival in relation to stage.

\section{DISCUSSION}

In our study, the proportion of ovarian malignancy cases was $5.92 \%$ of all gynaecological admissions during twoyear period. The proportion of ovarian malignancy was $48.8 \%$ among all genital malignancies while epithelial ovarian carcinoma (EOC) comprised $44.5 \%$ cases, thus contributing to $91 \%$ of all ovarian malignancies. Se Ik Kim et al reported histologic subtype in $90 \%$ cases as EOC. ${ }^{4}$ Basu et al reported $80 \%$ EOC among all ovarian malignancies while in study by Yogambal et al epithelial tumors were seen in $71.6 \%$ cases. ${ }^{1,5}$ Mondal et al reported EOC in $60.9 \%$ cases. $^{6}$ 
Various studies in literature reported increasing trends in ovarian cancer over the years., ${ }^{2,7-9}$ Murthy et al reported mean annual percent increase of ovarian cancer in India ranging from $0.7-2.4 \%$ in different age groups. ${ }^{2}$ This increasing trend may reflect increased exposure to risk factors or due to increased awareness and increase in proportion of elderly women.

Age has a strong corelation to ovarian cancer risk and $80 \%$ cases are diagnosed after 50 years of age. ${ }^{7}$ Advancing age increased the possibility of malignant transformation. Murthi et al reported that the disease increases from 35 years of age and reaches a peak between the ages 55-64. ${ }^{2}$ In their study, the mean age at diagnosis reported varied between 52.2 to 59.5 years. Saini et al in their study reported median age as 55years with mean age of $55.98 \pm 9.24$ years. $^{3}$ Basu et al reported mean age as $48.8 \pm 11.2$ years. ${ }^{1}$ Another study in India by Mondal et al reported median age of 48 years at diagnosis and maximum incidence of $44.3 \%$ in age group of $41-50$ years. ${ }^{6}$ An epidemiologic risk prediction model By $\mathrm{K}$ li reported median age of EOC in various countries as 52.4 years. ${ }^{8}$ In our study the mean age at diagnosis was $52.1 \pm 8.96$ years (median $=52$ ). Doufekas $\mathrm{K}$ reported mean age at diagnosis to be 63 years in UK. ${ }^{7}$

The mean age increased in relation to stage of EOC at diagnosis from $44 \pm 9.53$ years in stage 1 to $55.35 \pm 9.74$ years in stage 4 in present study. Similar findings were reported by Saini et al with mean age of $52.67 \pm 8.04$ in stage 1 to $58.30 \pm 8.48$ years in stage $4 .^{3}$ In a limited resource setting, ovarian malignancy screening program may be restricted to age group above 45 years, being high risk.

Nulliparity is reported as a risk factor in various studies. ${ }^{3,7-}$ ${ }^{9}$ The use of ovulation inducing agents in etiology of EOC is controversial ${ }^{7}$. There is $40 \%$ decrease in epithelial ovarian cancer after first live birth and risk reduction of about $14 \%$ for each subsequent pregnancy. ${ }^{9}$ In present study, $10.5 \%$ of ovarian malignancy patients were nulliparous though increasing parity did not show decrease in ovarian cancer. In the study by Saini et $\mathrm{al}^{3} 19.63 \%$ cases were nulliparous.

Genetic susceptibility is another risk factor reported. ${ }^{7}$ Approximately $10 \%$ cases are caused by mutations which cluster in families. Communicaion by Murthi et al reported that family history was present in only $4-5 \%$ cases. In our study family history was present in $6.1 \%$ cases. $^{2}$

Early menarche or late menopause is not shown to have correlation with epithelial ovarian cancer though McLemore reported association of late natural menopause with increased risk of EOC. ${ }^{7-9}$ Hormone Replacement Therapy (HRT) has shown a small risk with more than 10 years of use. ${ }^{7}$ In present study $65 \%$ cases were postmenopausal. Other factors which are related to increased risk include obesity due to increase in free biologically active estradiol, endometriosis (related to endometroid and clear cell carcinoma), asbestos and talc exposure. ${ }^{4}$ The protective factors include pregnancy, use of contraceptive pills, Non-steroidal anti-inflammatory drugs (NSAIDs), hysterectomy, and salpingooophrectomy. ${ }^{8,9}$ Tubal ligation decreased risk by $39 \%$ while Hysterectomy decreased by $50 \% .{ }^{9}$ Previous history of breast cancer and radiotherapy treatment also carried long term risk of ovarian cancer. ${ }^{7}$ The role of diet, ovulation inducing agents, ethnicity and smoking is inconclusive as the findings are contradictory in various studies. ${ }^{7-9}$ We could not comment on these factors as ours was retrospective study and data was missing from case records.

Clinical symptoms included dyspepsia, pain in abdomen and abdominal distension in majority of cases. Mclemore et al reported ovarian cancer symptom index (OCSI) consisting of bloating, pelvic or abdominal pain, feeling full quickly and urinary symptoms of urgency and frequency. ${ }^{9}$ If any of these symptoms were reported $>12$ times in one month, OCSI was positive with sensitivity of $56.7 \%$ to detect early stage disease and $79.5 \%$ for detection of advanced stage disease. Yogambal et al also reported pain abdomen $(66.92 \%)$ and mass in abdomen $(28.11 \%)$ as the commonest presenting symptom. ${ }^{5}$ Dyspepsia was most common complaint reported by Saini et al in $66.26 \%$ cases. $^{3}$ Patients present with dyspepsia to primary care physicians; therefore it is advisable that pelvis ultrasonography be done in elderly patients to detect disease in early stage. ${ }^{3}$ Post-menopausal bleeding is another symptom which warrants screening as it indicates presence of available circulating estrogen. ${ }^{9}$

The disease is diagnosed in late stages as there is delay between onset of symptoms and diagnosis. Doufekas and Olaitan reported that $60 \%$ cases were diagnosed in stage III and IV. ${ }^{7}$ In our series, $56 \%$ patients were in stage III and $31.4 \%$ were in stage IV. In study by Saini et al $20.8 \%$ cases were in stage II, $47.85 \%$ in stage III and $16.56 \%$ in stage IV. ${ }^{3}$ Doufekas et al reported $30 \%$ cases in stage II and $60 \%$ in stage III. Mondal et al had $20 \%$ cases in stage II and $60 \%$ in stage III while Basu et al reported $80 \%$ patients in stage III/IV at diagnosis. ${ }^{7,6,1}$

Determination of histology pattern is useful in diagnosis, treatment and prognosis in EOC. ${ }^{4,5}$ Endometroid carcinoma is identified at early stage and being chemosensitive is associated with better prognosis. Clear cell carcinoma when identified at early stage has good prognosis but in later stage has worse prognosis in comparison to serous carcinoma as it is less sensitive to platinum based chemotherapy. ${ }^{4}$ There is increase in clear cell carcinoma which is related to increased incidence of endometriosis while Mucinous carcinoma is related to ciggarette smoking. ${ }^{4}$

Commonest histopathological malignancy in all studies ${ }^{1,36}$ is serous carcinoma. Basu-41.6\%, Saini-49.69\%, Se le Kim-49.5\%, Yogambal-45\%, Mondal 38.3\%. ${ }^{1,3-6}$ Doufekas reported serous subtype in one third cases. ${ }^{7}$ Its 
malignant potential is highest, spread is faster but response to chemotherapy is good. In our study $85.5 \%$ cases were serous carcinoma. Hstopathological grading is also related to patient survival. Basu et al reported tumors to be well differentiated in $27 \%$, moderately differentiated in $48 \%$ and poor differentiation in $25 \%$ cases while in our study only $7 \%$ were well differentiated, $42 \%$ had moderate differentiation and $51 \%$ poor differentiation. ${ }^{1}$

The standard management consists of surgical staging with optimal cytoreduction followed by a platinum based chemotherapy as per stage of tumor. In present series, 35\% patients were treated with surgery i.e. Total Abdominal Hysterectomy (TAH) with Bilateral Salpingo Oophrectomy (BSO) with infracolic omentectomy, followed by chemotherapy. $20 \%$ patients were given $\mathrm{Neo}$ adjuvant chemotherapy (NACT), surgery and postoperative chemotherapy. $14 \%$ cases received palliative chemotherapy.

There is improvement in survival rates in early stage disease due to advancement in chemotherapy and surgery but unfortunately majority of the patients report in late stage. According to Basu et al optimal debulking could be done in only $20.3 \%$ cases due to advanced disease stage. Stage 4 patients were not treated with surgery in his study. ${ }^{1}$

Prognosis with ovarian cancer is poor with overall 5-year survival rates below $45 \% .^{7}$ Age at diagnosis, FIGO stage, histology, and histologic grade are associated with survival rates.

Doufekas reported 5-year survival rate of $84.2 \%$ in $15-39$ years while only $13.7 \%$ in women over 85 years of age. ${ }^{7}$ National statistics from Canada also reported similar findings of five-year survival in those aged 15-39 as 84\% compared with $14 \%$ in those aged 85 or over. ${ }^{10}$ In our study by multivariate analysis, age was found to be independent prognostic factor with $\mathrm{p}$ value of 0.004 . Stage of the disease also is an independent risk factor for poor prognosis with $\mathrm{p}$ value of .005 in our study. Other authors also reported poor survival with advancing disease stage.

Basu et al reported 5-year survival as $85 \%$ for stage I, 71\% for stage I, $41 \%$ for stage III and $22 \%$ for stage IV. ${ }^{1}$ Saini et al reported poor survival with advancing stage of disease using Kaplan Meier curves with log rank of $\mathrm{P}<0.05$. $^{3}$ FIGO Stage IV showed poor survival in comparison to Stage I tumors (log-rank $P<0.0001)$, Stage II (log-rank $P$ $=0.0003)$, and Stage III $(P=0.0041)$. Stages II and III had poor survival in comparison to Stage I (log-rank $P=$ 0.0280 and 0.0011$)$, but no difference in stages II and III (log-rank $P=0.1830$ ). Yogambal et al reported 5-years survival rates for tumor stage I-85\%, stage II-71\%, stage III-41\% and stage IV-22\% respectively. ${ }^{5}$ Mondal et al also reported similar 5-year survival rates as $85 \%$ for stage I, $65 \%$ for stage II, $30 \%$ for stage III and $15.5 \%$ for stage IV. ${ }^{6}$ Doufekas reported 5-year survival rate in various stages from I to IV as $80 \%, 30 \%, 22 \%$ and $5.6 \%$. $^{7}$
In their study one third women died within one year of diagnosis indicating disease presentation in advanced stage. They also reported improvement in survival rates in stage I and II over 20 years.

Survival was not significantly different in various histopathology subtypes in our study log rank $\mathrm{P}=0.629$ and by multivariate analysis $\mathrm{P}=0.688$. Similar findings were reported by Saini et al $(\log$-rank $P=0.7406) .^{3}$

\section{CONCLUSION}

Epithelial ovarian cancer has emerged as one of the commonest malignancy affecting women in India. A steady increase has been observed in the incidence of ovarian cancer in several registries.

EOC is disease of fifth decade and above age group. Its diagnosis is done in advanced stage with poor survival.

Efforts should be made to detect the disease at early stage through population education with respect to epidemiological factors. Currently, a major goal of ovarian cancer research is to develop an effective test that can detect the disease at its earliest stages, which would ultimately result in decreased mortality.

Increased knowledge of ovarian cancer etiology and pathogenesis would greatly enhance the development of this tool.

Another approach that can improve diagnosis of epithelial ovarian cancer is to educate primary care physician about ovarian cancer and to include it in differential diagnosis in the specific patient population.

Further studies are needed to elicit the causative factors responsible for the increase in the incidence of the disease and also their mechanism of action. Most ovarian cancers are environmental in origin and therefore in principle preventable.

It is suggested that in low resource settings like India, an epidemiological study should be done to find effect of screening programs using relatively easy available imaging modality (USG) on diagnosis in ovarian cancer risk age group patients with vague gastric symptoms.

\section{ACKNOWLEDGMENTS}

Authors would like to thanks Dean Goa Medical College for allowing us to use hospital data for preparation of this manuscript.

Funding: No funding sources

Conflict of interest: None declared

Ethical approval: The study was approved by the Institutional Ethics Committee 


\section{REFERENCES}

1. Basu P, De P, Mandal S, Ray K, Biswas J. Study of 'patterns of care' of ovarian cancer patients in a specialized cancer institute in Kolkata, eastern India. Indian J Cancer. 2009;46:28-33.

2. Murthy NS, Shalini S, Suman G, Pruthvish S, Mathew A. Changing Trends in Incidence of Ovarian Cancerthe Indian Scenario. Asian Pacific J Cancer Prev. 2009;10:1025-30.

3. Saini SK, Shrivastava S, Singh Y, Dixit AK, Prasad SN. Epidemiology of epithelial ovarian cancer, a single institution based study in India. Clin Cancer Investig J. 2016;5:20-4.

4. Kim SI, Lim MC, Lim J, Won YJ, Soo S, Kang SS, et al. Incidence of epithelial ovarian cancer according to histologic subtypes in Korea. 1999 to 2012. J Gynecol Oncol. 2016;27(1):5.

5. Yogambal M, Arunalatha P, Chandramouleeswari K, Palaniappan V. Ovarian tumours- Incidence and distribution in a tertiary referral center in south India. IOSR. 2014;13(2):74-80.

6. Mondal SK, Banyopadhyay R, Nag DR, Roychowdhury S, Mondal PK, Sinha SK. Histologic pattern, bilaterality and clinical evaluation of 957 ovarian neoplasms: A 10-year study in a tertiary hospital of eastern India. J Can Res Ther. 2011;7:4337.

7. Doufekas K, Olaitan A. Clinical epidemiology of epithelial ovarian cancer in the UK. International Journal of Women's Health 2014:6 537-45.

8. Li K, Husing A, Fortner R, Tjønneland A, Hansen L, Dossus L, et al. An epidemiologic risk prediction model for ovarian cancer in Europe: the EPIC study. British J Cancer. 2015;112:1257-65.

9. Monica R. McLemore, Christine Miaskowski, Bradley E. Aouizerat Lee-may Chen, Marylin J. Dodd. Epidemiologic and Genetic Factors Associated with Ovarian Cancer Cancer Nurs. 2009; 32(4):28190 .

10. Statistics Canada, Catalogue no. 82-624-X. Health at a Glance: 2015.

Cite this article as: Jindal D, Sahasrabhojanee M, Jindal M, D'Souza J. Epidemiology of epithelial ovarian cancer: a tertiary hospital based study in Goa, India. Int J Reprod Contracept Obstet Gynecol 2017;6:2541-6. 